\title{
Transactions Papers
}

\section{Orthogonal Frequency-Division Multiplex Transmission of H.263 Encoded Video over Highly Frequency-Selective Wireless Networks}

\author{
Peter Cherriman, Thomas Keller, and Lajos Hanzo, Senior Member, IEEE
}

\begin{abstract}
The video performance of a 155 -Mbps wireless asynchronous transfer mode (WATM) proposal and that of a 2-Mbps Universal Mobile Telecommunications System (UMTS) concept is evaluated for a range of low- to high-quality video application scenarios, various propagation conditions, and video bit rates using the $\mathbf{H . 2 6 3}$ video codec, assisted by a novel packetization and packet acknowledgment scheme. Orthogonal frequency-division multiplexing is invoked over the highly dispersive channels for conveying high-rate video signals. Various binary Bose-Chaudhuri-Hochquenghem and turbo codes are investigated comparatively, with the conclusion that due to the high error resilience of the video packetization and acknowledgment scheme, the increased power of the higher complexity turbo codec does not translate to substantially improved overall system robustness, although the bit error rate and acknowledgment flag error rate are significantly reduced. The whole range of video resolutions and system parameters is summarized for reasons of space economy in Tables II-IV. The required channel signal-to-noise ratio for near-unimpaired video quality is about $16 \mathrm{~dB}$ for the inherently lower quality, lower resolution video frame formats, but slightly higher, about $18 \mathrm{~dB}$, for the high-definition formats, where the error-induced subjective video degradations become more objectionabl over the highly dispersive worst case channels used.
\end{abstract}

Index Terms-H.263-based wireless video communications, orthogonal frequency-division multiplexing (OFDM)-based video.

\section{BACKGROUND ${ }^{1}$}

W HILE the penetration of second-generation voice- and data-oriented wireless systems is increasing at an unprecedented rate, the wireless communications community is studying a range of third-generation standard proposals, which are summarized, for example, by Ojanpera in [1]. In Europe, these initiatives are managed under the auspices of the socalled Universal Mobile Telecommunications System (UMTS)

Manuscript received November 20, 1998. This work was supported by Motorola ECID, Swindon, U.K., by the European Commission under the Median project, and by EPSRC, U.K. This paper was recommended by Associate Editor J. Brailean.

The authors are with the Department of Electronics and Computer Science, University of Southampton, Southampton SO17 1BJ U.K. (e-mail: 1h@ecs.soton.ac.uk).

Publisher Item Identifier S 1051-8215(99)06182-0.

${ }^{1} \mathrm{~A}$ range of video demonstrations supporting this treatise can be found at http://www-mobile.ecs.soton.ac.uk/peter/robust-h263/robust.html.
[2] initiative, and at the time of writing, a number of standard proposals have been submitted to the European Telecommunications Standardization Institute [1]. Specifically, wideband code-division multiple access (CDMA) is advocated by Nokia from Finland and the Pan-European Frames UMTS consortium, which was documented, for example, by Pehkonen et al. [3]. Adaptive time-division multiple access (TDMA) [4] is a system framework, which was originally proposed in the European Research in Communications Equipment (RACE) program [2], [5], the predecessor of the current Advanced Communications Technologies and Services (ACTS) program [6], [7]. The remaining proposals are based on hybrid TDMA/CDMA, advocated by Baier et al. from Kaiserlautern University [8]; orthogonal frequency-division multiplex (OFDM) suggested by Telia in Sweden [9], [10], which is studied in this paper; and opportunity-driven multiple access (ODMA). Most proposals were formulated with multimedia capabilities, including interactive video communications, in mind, as evidenced for example by the Nokia testbed [11] or by the Japanese wide-band CDMA proposal by Adachi [12] for the intelligent mobile terminal IMT 2000 emerging from NTT DoCoMo. Most of these proposals were targeted at highdispersion cellular environments, where high excess delay path lengths prevail, which limit the maximum bit rate supported to around $2 \mathrm{Mbps}$. Hence in one of our candidate systems here, we will study the video performance of an OFDMbased UMTS-oriented frames-like scheme at a rate around 2 Mbps using the system parameters of Table IV, which will be detailed during our forthcoming elaborations.

In the field of wide-band communications, the developments have been equally rapid [13]-[18], leading to a variety of wireless local-area network (WLAN) proposals [16]-[18]. Similarly to the above-mentioned OFDM-based UMTS-type systems [19], OFDM had been found advantageous for combating dispersion in WATM networks [20]. Hence in our second candidate system, we will evaluate the ability of OFDM to support high-rate, high-quality WATM video communications at bit rates around $20 \mathrm{Mbps}$ under various conditions, which are summarized in Tables II and IV that will be discussed. 
TABLE I

Video Seouences UsEd For H.263 Simulations

\begin{tabular}{l|l|c|c}
\hline Video Clip & Size & Frame/s & Colour \\
\hline Miss America & QCIF & 10,30 & Grey \\
Miss America & SQCIF, QCIF, CIF & 10,30 & Colour \\
Carphone & QCIF & 10,30 & Colour \\
Suzie & SQCIF, QCIF, 4CIF & 10,30 & Colour \\
Football & 4CIF & 10,30 & Colour \\
Mall & 16CIF & 10,30 & Colour \\
CIF: 288x352 pixel Common Intermediate Format \\
QCIF: 144x176 pixel Quarter CIF \\
SQCIF: 96x128 pixel Sub-QCIF \\
4CIF: 576x704 pixel 4xCIF \\
16CIF: $1152 \times 1408$ pixel 16xCIF
\end{tabular}

OFDM was originally proposed by Chang in 1966 [21], where instead of estimating the wide-band dispersive channel's impulse response, as in conventional equalized serial modems [10], the channel is rendered nondispersive by splitting the information to be transmitted in a high number of parallel, lowrate, nondispersive channels [10]. In this case, there is no need to estimate the channel's impulse response, since for the lowrate subchannels, it can be considered nondispersive. Over the past three decades this technique has been regularly revisited by a number of researchers [21]-[26]. Despite its conceptual elegance, until recently, its employment has been mostly limited to military applications due to implementational difficulties. However, it has recently been adopted as the new European digital audio broadcasting (DAB) standard, and it is also a strong candidate for digital terrestrial television broadcast (DTTB) and for a range of other high-rate applications, such as 155 -Mbps wireless asynchronous transfer mode (WATM) LAN's. These wide-ranging applications underline its significance as an alternative technique to conventional channel equalization in order to combat signal dispersion [21]-[26].

The associated video-compression issues have been the topic of the monographs by Netravali and Haskell [27], Jain [28], Jayant and Noll [29], etc. Khansari et al. [31], as well as Pelz [32], contrived appropriate transmission schemes for the H.261 codec, while Faerber et al. [33] proposed transmission schemes for the H.263 codec [34]. Gharavi and Partovi [35] proposed an attractive video communications scheme for broad-band networks. Further serial-modem-based video transceivers were proposed in [36] and [37]. The most important recent development in the field of video compression is hallmarked by the MPEG-4 standardization activities [43].

The outline of this paper is as follows. In Section II, the ability of the H.263 codec to support a wide range of video services is analyzed. Section III provides a system overview, while Section IV characterizes the propagation environment of both our UMTS-like and WATM-oriented systems. Section V is concerned with the system's video aspects, and the overall system performance is evaluated in Section VI.

\section{Performance of the H.263 Codec}

In this section, we briefly analyze the image quality versus bit rate performance of the H.263 codec for various image resolutions, frame rates, and video sequences. The video sequences used are summarized in Table I. We note, however, that for cellular and cordless systems, only SQCIF and QCIF resolutions are realistic in terms of their minimum required bit rates, while for higher rate LAN's, CIF, 4-CIF, and 16-CIF resolutions cater to substantially increased video quality. The corresponding peak signal-to-noise ratio (PSNR) results are portrayed in Fig. 1 as a function of bit rate and compression ratio, respectively, when using the Miss America sequence. The quadrupled number of pixels present in the CIF format resulted in an approximately four-fold increase of the bit rate. Observe, furthermore, that the 30 frames per second (fps) scenarios required typically a factor of two higher bit rates in order to maintain a certain fixed PSNR.

A range of further similar curves were generated using video sequences of various resolution, of which we present here various high-resolution, 4-CIF, and 16-CIF results. As before, our experiments were carried out at 10 and $30 \mathrm{fps}$. The 4-CIF Suzie video sequence, the 4-CIF American Football sequence, and the high-definition 16-CIF Mall video sequence were used in these experiments. The associated performance curves are shown in Fig. 2. Since the amount of image fine detail is quite different in the Suzie, American Football, and Mall sequences, the corresponding performance curves are not strictly comparable, but they give an estimate of the expected bit rates in relative terms. As can be seen for the graphs in Fig. 2, the 4-CIF American Football has similar performance to the 16-CIF Mall sequence, primarily due to the large amount of motion in the American Football sequence. For a more detailed exposure to the H.263 codec, the interested reader is referred to [44]. Let us now consider our transmission schemes in the next section.

\section{SYSTEM OVERVIEW}

Both proposed wireless systems' schematics follow the structure of Fig. 3, and both support interactive videophone calls. The video signal is compressed using the H.263 videocompression standard [34], as discussed in Section II. The H.263 standard achieves a very high compression ratio; however, the resulting bitstream is extremely sensitive to channel errors. This sensitivity to channel errors is not a serious problem over benign wireline-based channels, such as conventional ATM links, but it is an impediment when used over wireless networks. There have been several solutions suggested in the literature for overcoming this using automatic repeat request (ARQ) [30], dual-level coding [31], and a feedback channel [33]. A range of further robust video schemes was proposed in [36]-[40].

As seen in Fig. 3, our system uses a feedback channel to inform the encoder of the loss of previous packets. However, we do not retransmit the corrupted packets, since this would reduce the system's teletraffic capacity by occupying additional transmission slots while increasing the video delay. We found that simply dropping the corrupted packets at both the local and the remote decoder results in an extremely high error resilience, in particular in high-frame-rate systems, where 30fps high-rate transmissions are facilitated. The rationale behind this is that nonupdated video frame segments can only persist at $30 \mathrm{fps}$ for $33 \mathrm{~ms}$. This allows for the reconstruction frame 


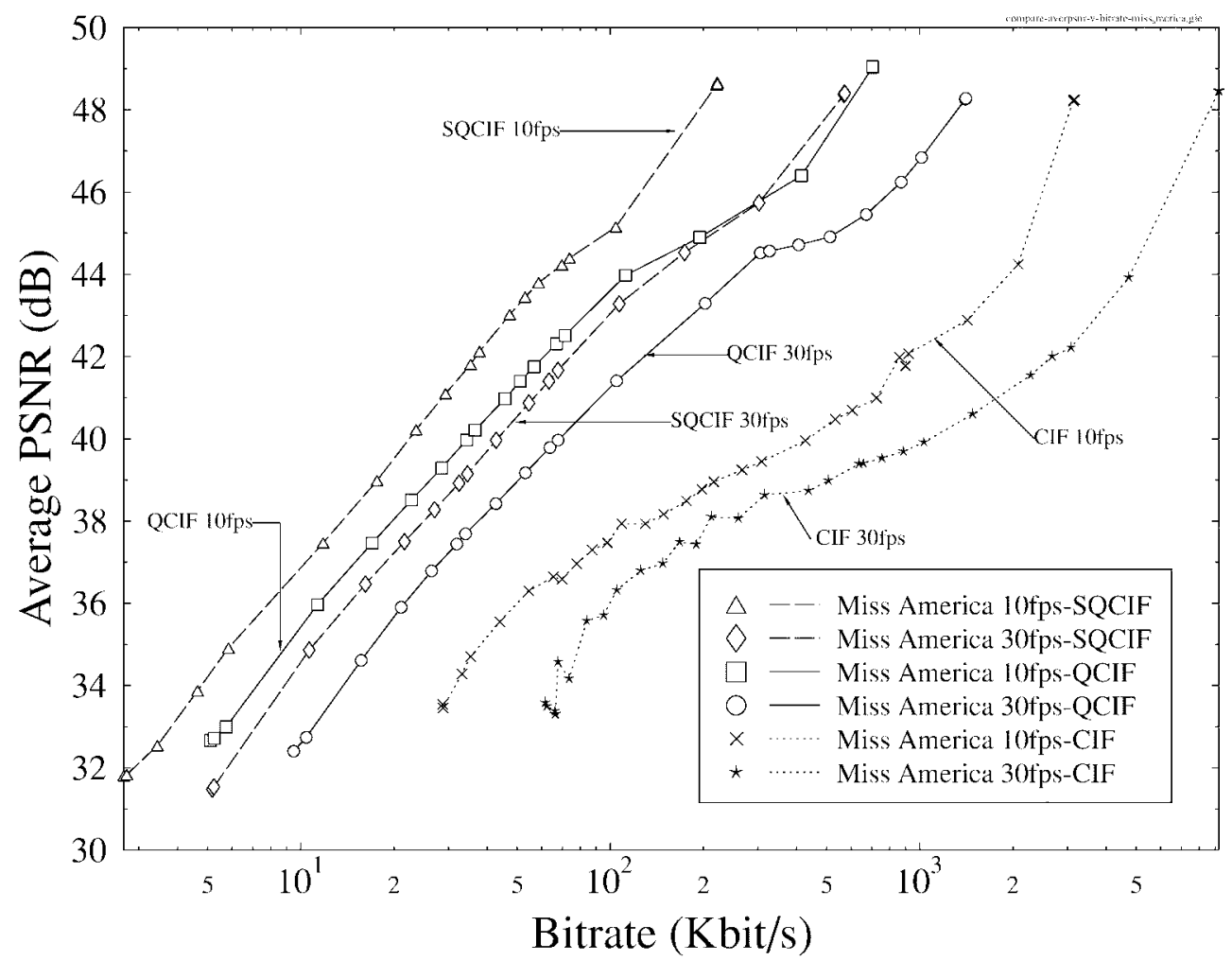

Fig. 1. Image quality (PSNR) versus coded bit rate for H.263 Miss America simulations at 10 and 30 frames/s.

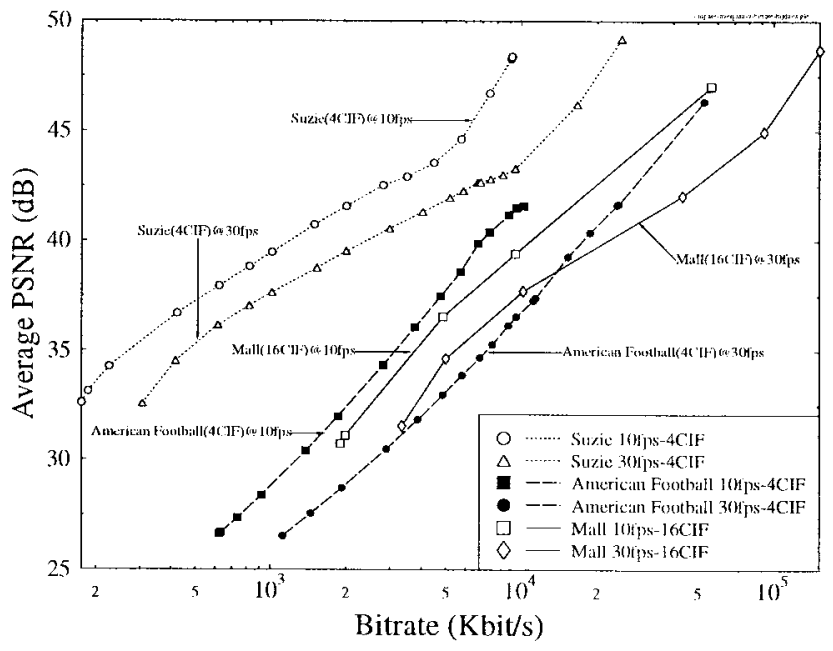

Fig. 2. Image quality (PSNR) versus coded bit rate for H.263 high-resolution (4 CIF and $16 \mathrm{CIF}$ ) simulations at 10 and $30 \mathrm{fps}$.

buffer contents of the local and remote decoders to remain identical, which is essential for preventing error propagation through the reconstructed frame buffer. Then, when the instantaneous channel quality improves, the corrupted picture segments of the reconstructed frame buffers are replenished with more up-to-date video information. The feedback channel is implemented by superimposing the packet dropping request on the reverse link, as shown in Fig. 4. This figure shows how the feedback acknowledgment is implemented in the context of the proposed TDMA, time-division duplex (TDD) system using 32 time slots, where one video packet was transmitted in each TDD frame. A number of further interesting details

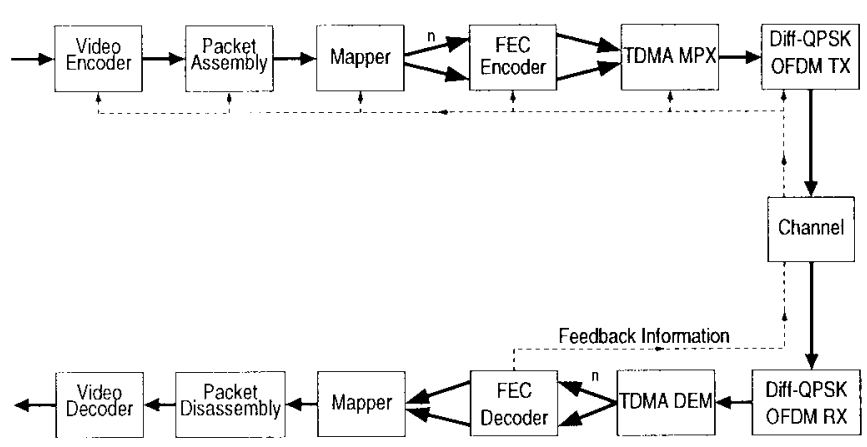

Fig. 3. Transceiver schematic.

concerning the system's operation become explicit by studying the figure in more depth, which we have to refrain from here due to lack of space.

As demonstrated by Fig. 3, the H.263 encoded bitstream in passed to the packet assembly block, which was detailed in [37]. The packetizer's function is to assemble the video packets for transmission, taking into account the packet acknowledgment feedback information. The packet disassembly block of the figure ensures that always an error-free H.263 bitstream is output to the video decoder, discarding any erroneously received packet and using only error-free packets to update the reconstructed frame buffer. Since the transmission packets contain typically fractions of video macroblocks at the beginning and end of the packets, a corrupted packet implies that the previously received partial macroblocks have to be discarded. The loss of the packet is then signalled via the feedback channel to the video encoder and packet assembly blocks. 


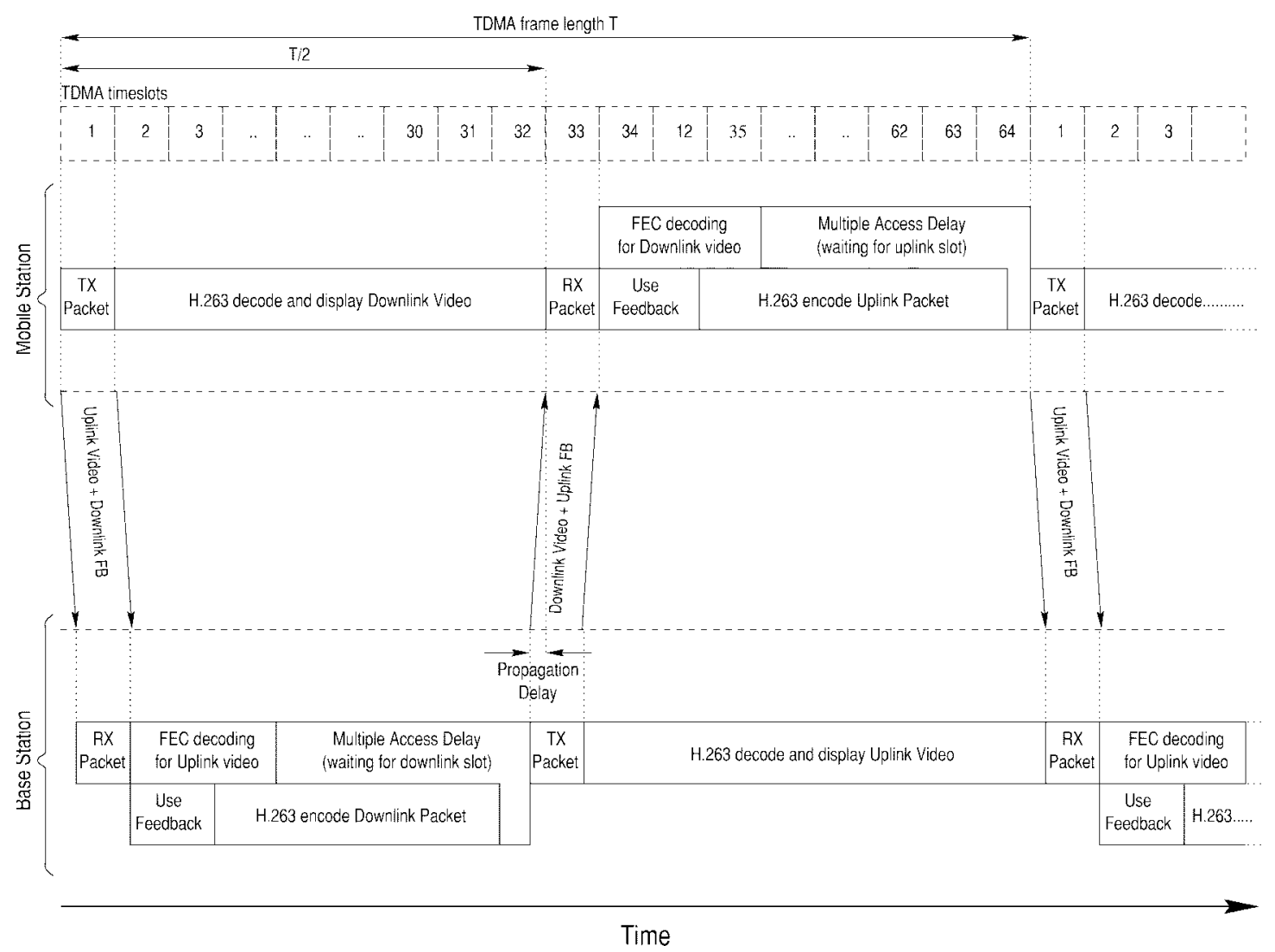

Fig. 4. Transmission feedback timing diagram showing the feedback signalling superimposed on the reverse channel video datastream. The tasks that need to be performed in each time interval are shown for both the mobile station and the base station.

The lost macroblocks are not retransmitted, but strongly error-protected acknowledgment flags are inserted into the video bitstream to signify the macroblocks that have not been updated. This requires one bit per lost macroblock in the next reverse-direction packet of the given user. The decoded video stream is error free, although certain parts of some video frames may be "frozen" for a frame's duration due to lost packets. These areas will be usually updated in the next video frame, and the effect of the lost packet will be no longer visible. Again, this packet loss has a prolonged effect for $100 \mathrm{~ms}$ at $10 \mathrm{fps}$, which is more objectionable than the losses at $30 \mathrm{fps}$. These operations were also indicated earlier in the context of Fig. 4, while aspects of the acknowledgement flag protection and the associated probability of correct flag reception are quantified in Figs. 5 and 14, which will be discussed at a later stage.

The packetized video stream is then forward error correction (FEC) coded, mapped to the allocated TDMA time slot, and transmitted using differential quarternary phase shift keying (D-QPSK) between adjacent subcarriers of the OFDM scheme employed [10]. Again, it is important to strongly protect the binary acknowledgment flag from transmission errors, which prevents the remote decoder from updating the local reconstruction buffer, if the received packet was corrupted. Following a range of considerations, we opted for using a repetition code, which was superimposed on the forthcoming reverse-direction packet in the proposed TDD scheme of Table II. The repetition-coded flag is then majority logic decision (MLD) decoded at the receiver. The probability of correct decoding of the 5-, 9-, 18-, and 27-bit majority logic codes was numerically evaluated for the range of bit error rates (BER's) $0-50 \%$ using a random error distribution in Fig. 5. On the basis of the results, we opted for using the strongest MLD code of $\operatorname{MLD}(27,1,13)$, repeating the flag 27 times, which was hence able to correct up to 13 transmission errors or a channel BER of about $50 \%$.

In the next section, we briefly consider the specific system parameters used, which closely resemble those proposed by the Pan-European Wireless Asynchronous Transfer Mode consortium, referred to as MEDIAN.

\section{A. The WATM System}

The system employed in our experiments resembles the ACTS MEDIAN WATM proposal, operating in the $60-\mathrm{GHz}$ band utilizing OFDM as a modulation technique [10]. The MEDIAN-like WATM system parameters are listed in Table II. Channel access in the MEDIAN WATM system is based on TDMA/TDD frames having a duration of $170.7 \mu \mathrm{s}$. This frame is split into 64 time slots of 2.667- $\mu$ s duration. Two of these time slots are reserved for networking functions, leaving 62 for useful information transfer.

To avoid implementationally complex equalization at the FEC-coded sampling rate, OFDM is employed as a baseband modulation technique [10]. D-QPSK between adjacent subcarriers is used as a frequency-domain modulation scheme. If all 512 subcarriers are used and one subcarrier is used as 


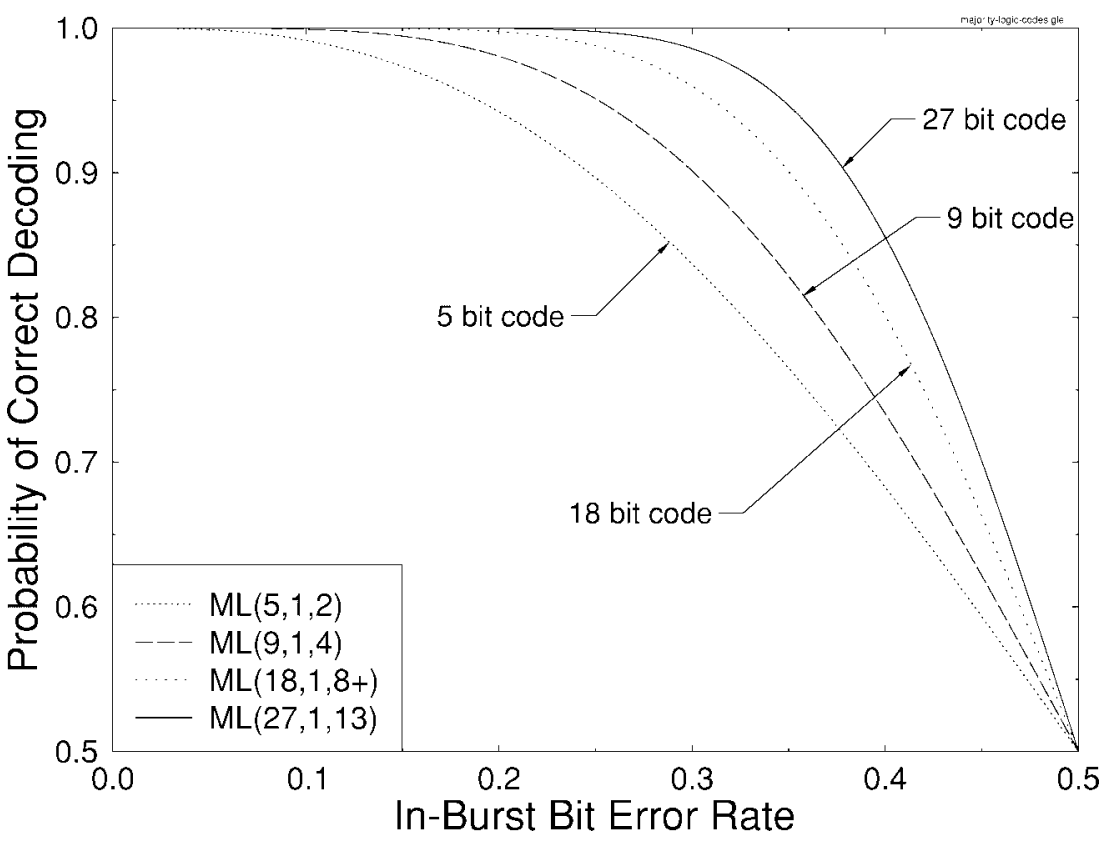

Fig. 5. Numerical evaluation of the probability of correct decoding $\left(P_{\mathrm{CD}}\right)$ of majority logic codes.

TABLE II

Summary of THE MEDIAN-LiKe WATM System Parameters

\begin{tabular}{l|l}
\hline Feature & Value \\
\hline TDMA/TDD frame length & $171 \mu \mathrm{s}$ \\
Slots/Frame & $64(61$ uscable) \\
Slot length & $2.667 \mu \mathrm{s}$ \\
OFDM carriers & $512(511$ used) \\
Modulation & Differential-QPSK \\
Coded Bits/slot & 1022 bits \\
FEC (1/2 rate) & BCH $(255,131,18)$ \\
Pre-FEC bits/slot & 524 bits \\
System Bandwidth & $225 \mathrm{MHz}$ \\
System Symbol rate (symbols/sec) & $186 \times 10^{6}$ \\
Normalised Doppler Frequency & $1.235 \times 10^{-5}$ \\
\hline
\end{tabular}

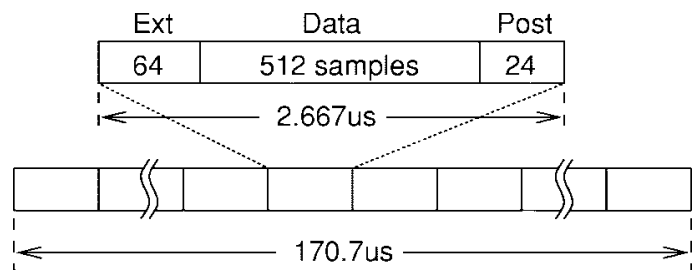

Fig. 6. Schematic plot of the ACTS MEDIAN WATM frame structure. A time frame contains 64 time slots of 2.667- $\mu$ s duration. Each time slot holds the data samples of a 512-point inverse fast Fourier transform (IFFT) OFDM symbol, 64 samples of the cyclic extension, and a cyclic postamble of 24 samples.

phase reference, then 1022 bits can be transmitted using a single OFDM symbol. Fig. 6 portrays the MEDIAN WATM frame and slot structure. Each time slot contains one OFDM symbol, preceded by a cyclic extension of 64 samples in order to combat interference in wide-band channels [10]. A cyclic postamble is appended to the OFDM data samples in order to simplify symbol timing synchronization [20]. For our simulations, all 512 subcarriers per OFDM symbol were used, resulting in the maximum throughput of 1022 bits per OFDM symbol.

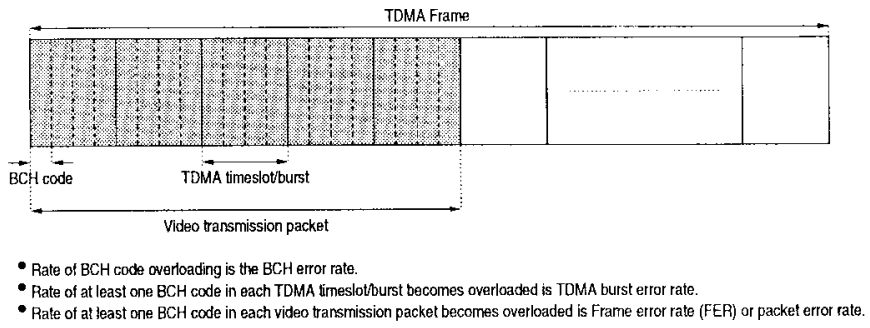

Fig. 7. Stylized TDMA frame structure for the WATM system transmitting 4-CIF-resolution video, where each video transmission packet is formed using five time slots per active TDMA frame and each time slot contains four Bose-Chaudhuri-Hochquenghem $(\mathrm{BCH})$ code words.

Variable-bit-rate users can be accommodated by allocating groups of time slots per frame, as seen in Table III, in case of high-rate users or by skipping time frames in case of low-rate users. The shaded area of Fig. 7 defines the "payload" of a TDMA frame in the 4-CIF scenario of the table, and hence this payload must be received free of error. If it is corrupted, this event is defined as a TDMA frame error, and the relative frequency of these events defines the frame error rate (FER) used in the system. As seen in Fig. 7 and Table III, in this case we need five time slots for supporting the associated 10.2-Mbps video rate, but other video resolutions require a different number of time slots. Their FER is defined on the basis of the success or failure of all the slots of a specific video user in a TDMA frame, since in this regime we cannot selectively retransmit the payload of each time slot due to having only one acknowledgment flag per TDMA frame per user. This results in larger video frame sections' remaining nonupdated due to the increased payload per TDMA frame at high video rates. Again, the required number of slots per TDMA frame for each video mode was summarized for the various modes in Table III, while the companion paper [45] proposed an efficient statistical 
TABLE III

SUMMARY OF VIDEO PARAMETERS FOR THE WATM SYSTEM

\begin{tabular}{l||c|c|c|c}
\hline \multicolumn{1}{l||}{ Feature } & \multicolumn{3}{c}{ Video Resolution } \\
\cline { 2 - 5 } & QCIF & CIF & 4 CIF & $16 \mathrm{CIF}$ \\
\hline \hline Luminance resolution (pixels) & $176 \times 144$ & $352 \times 288$ & $704 \times 576$ & $1408 \times 1152$ \\
\hline Crominance Resolution (pixels) & $88 \times 72$ & $176 \times 144$ & $352 \times 288$ & $704 \times 576$ \\
\hline Packet separation (in No. of TDMA frames) & 30 & 6 & 1.5 & 1 \\
\hline Packet rate (packets/s) & 195 & 975 & 3900 & 5448 \\
\hline Bits/Timeslot & 1022 & 1022 & 1022 & 1022 \\
\hline Timeslots per active TDMA frame & 5 & 2 & 5 & 7 \\
\hline Bits per active TDMA frame (packet size) & 5110 & 2044 & 5110 & 7154 \\
\hline Channel Bitrate & $1 \mathrm{Mbps}$ & $2 \mathrm{Mbps}$ & $20 \mathrm{Mbps}$ & $41.8 \mathrm{Mbps}$ \\
\hline FEC & $20 \times \mathrm{BCH}(255,131,18)$ & $8 \times \mathrm{BCH}(\ldots)$ & $20 \times \mathrm{BCH}(\ldots)$ & $28 \times \mathrm{BCH}(\ldots)$ \\
\hline Pre-FEC Bits per active TDMA frame & 2620 & 1048 & 2620 & 3668 \\
\hline Pre-FEC Bitrate & $511 \mathrm{Kbps}$ & $1 \mathrm{Mbps}$ & $10.2 \mathrm{Mbps}$ & $21.5 \mathrm{Mbps}$ \\
\hline Feedback control bits & 26 & 24 & 26 & 29 \\
\hline H.263 Packetisation header bits & 13 & 12 & 13 & 14 \\
\hline Video bits per active TDMA frame & 2581 & 1012 & 2581 & 3625 \\
\hline Useful Video Bitrate & $503 \mathrm{Kbps}$ & $1 \mathrm{Mbps}$ & $10 \mathrm{Mbps}$ & $21.2 \mathrm{Mbps}$ \\
\hline
\end{tabular}

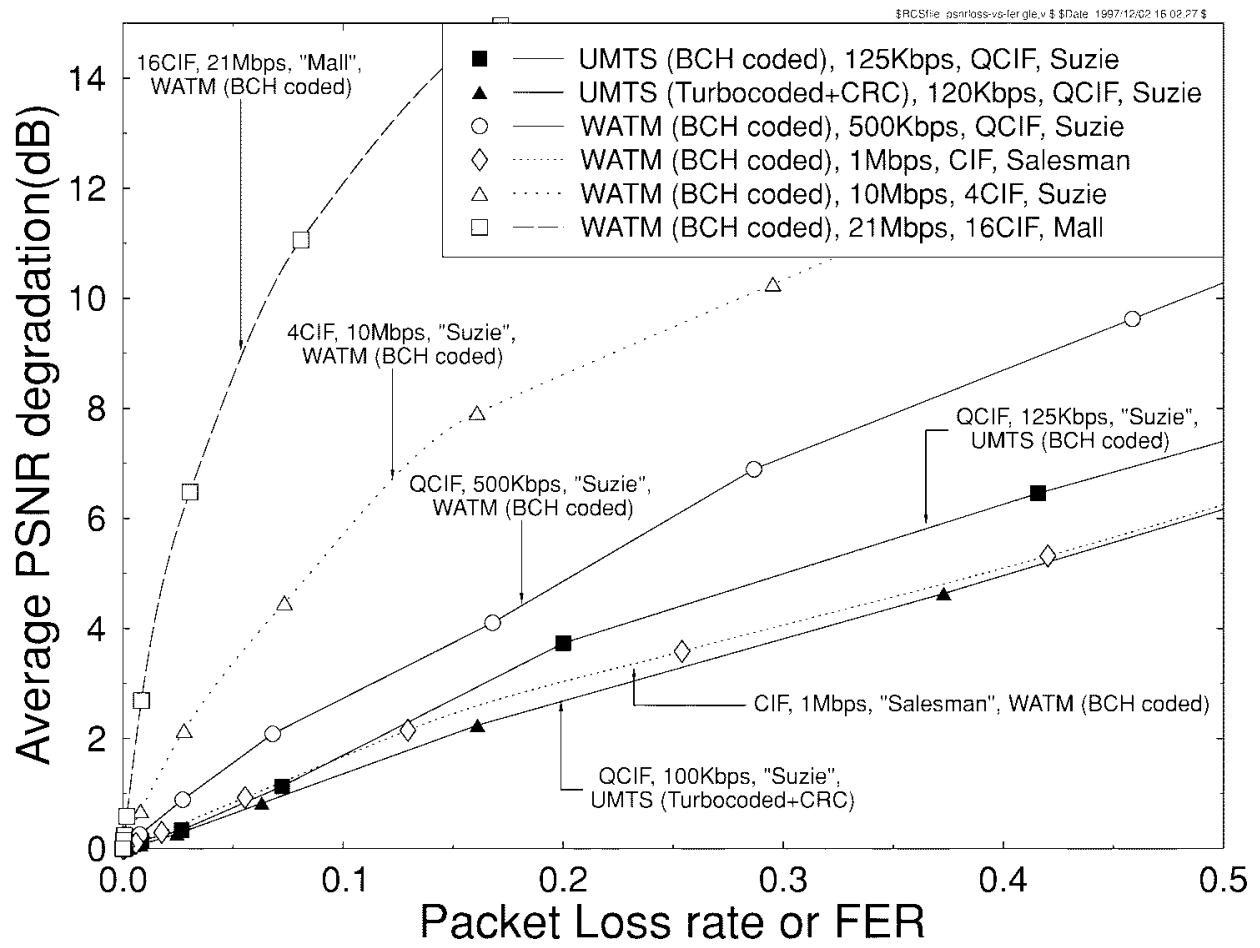

Fig. 8. PSNR degradation versus video packet loss rate or transmission frame error rate for the scenarios described in Tables III and IV.

multiplexing scheme for variable-rate scenarios, where the number of slots can be varied on a slot-by-slot basis. The associated PSNR degradation of the various user scenarios of Table III was quantified in Fig. 8, where the more dramatic PSNR degradation of the larger video frame sizes becomes explicit for any given transmission frame error rate.

\section{B. The UMTS-Type Framework}

The alternative transmission scheme used in our investigations was partially inspired by the ACTS FRAMES [3], [11] Mode 1 proposal, which entails a time-frame structure of $4.165 \mathrm{~ms}$, split into eight time slots of 577- $\mu$ s duration each. However, instead of using the originally proposed DS-CDMA scheme with a chip rate of $2.17 \mathrm{Mchips} / \mathrm{s}$, we have employed
1024 subcarrier OFDM, as shown in Fig. 9. Hence we refer to this system as a FRAMES-like scheme. The modified time slot contains a 1024-sample OFDM symbol, which is preceded by a cyclic extension of 168-samples length and followed by a guard interval of 60 samples. To maintain the FRAMES UMTS bandwidth of $1.6 \mathrm{MHz}$, the 1024 subcarrier OFDM symbol contains 410 virtual subcarriers, therefore reducing the bandwidth to $1.3 \mathrm{MHz}$ and allowing for a modulation excess bandwidth. Let us now consider the corresponding channel models.

\section{ThE Channel Model}

The channel model employed for the WATM system experiments was a five-path, Rayleigh-fading indoors channel. 


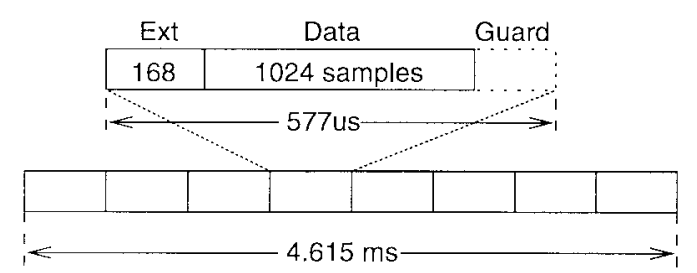

Fig. 9. Schematic plot of the ACTS FRAMES Mode-1-like frame structure as used in this paper. A time slot of 4.165-ms duration is split into eight time slots of $577 \mu \mathrm{s}$. Each time slot holds a 1024-point IFFT OFDM symbol with a cyclic extension of 168 samples.

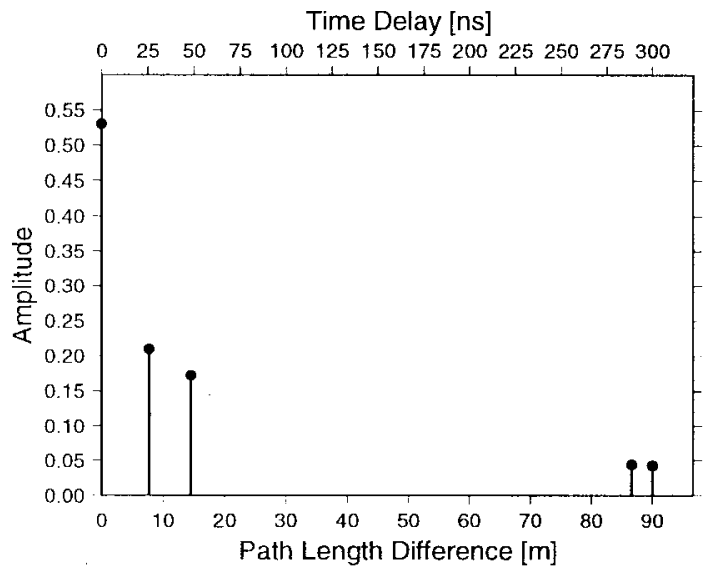

Fig. 10. WATM five-path impulse response.

The impulse response shown in Fig. 10 was obtained by ray tracing for a $100 \times 100 \mathrm{~m}^{2}$ hall or warehouse environment, and every path in the impulse response was faded independently according to a Rayleigh fading narrow-band channel with a normalized Doppler frequency of $f_{d}^{\prime}=1.235 \cdot 10^{-5}$, corresponding to the $60-\mathrm{GHz}$ propagation frequency and a worst case indoor speed of $30 \mathrm{mph}$.

A transmission rate of $155 \mathrm{Mbps}$ was used, which is applicable to WATM systems. A seven-path channel, corresponding to the four walls, ceiling, and floor plus the line-of-sight (LOS) path, was employed. The LOS path and the two reflections from the floor and ceiling were combined into a single path in the impulse response. The worst case impulse response associated with the highest path length and delay spread was experienced in the farthest corners of the hall, which was determined using inverse second-power law attenuation and the speed of light for the computation of the path delays. The corresponding frequency response was plotted in Fig. 11 for a 128-channel system as a function of both the time-domain OFDM symbol index and the frequency-domain subchannel index. Observe the very hostile frequency-selective fading in the figure, which is efficiently combated by the OFDM modem, since for each of the 512 narrow subchannels the channel can be considered more or less flat fading. The residual frequency-domain subchannel transfer function "tilt" or distortion can be equalized using a simple frequencydomain pilot-assisted equalizer.

The channel model used for the UMTS-type system experiments was based on a COST 207 [46] Bad Urban conformant

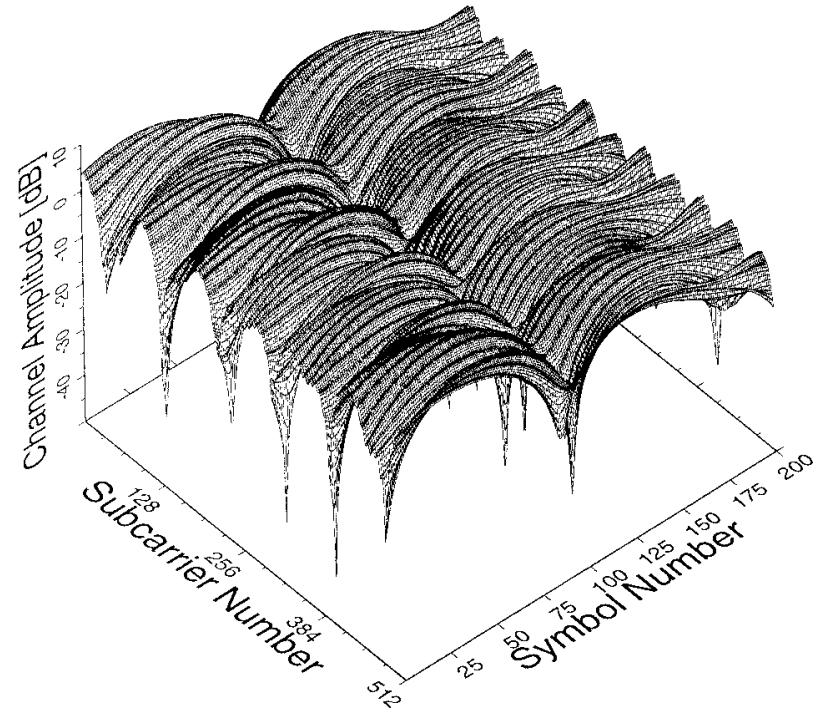

Fig. 11. Frequency response of the 512-subcarrier WATM OFDM system at 155 Mbps.

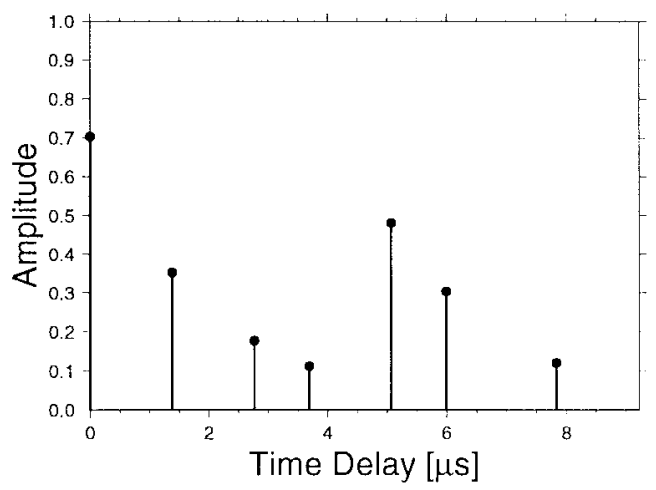

Fig. 12. COST 207 Bad Urban compliant impulse response, used for the UMTS [46].

seven-path impulse response shown in Fig. 12. Again, each of the impulses was faded independently according to a Rayleigh narrow-band fading channel with a normalized Doppler frequency of $f_{d}^{\prime}=92.6 \mathrm{~Hz} / 2.17 \mathrm{MHz}=4.267 \cdot 10^{-5}$, where the carrier frequency and vehicular velocity were set to $2 \mathrm{GHz}$ and $30 \mathrm{mph}$, respectively.

\section{VidEO-RELATED SYSTEM ASPECTS}

\section{A. Video Parameters of the WATM System}

Our high-rate WATM system constitutes an ideal medium for high-resolution video transmission. To assess the system's ability to support various application scenarios, we investigated four video systems of different resolution, ranging from QCIF to 16-CIF frame formats, as seen in Table III. The video packetizer operated most efficiently when the transmission packet-generation rate was neither too high nor too low. If the packet-generation rate per TDMA frame is too high, each packet may contain less than a whole macroblock, leading to an increased buffering in the depacketizer [37]. If the packet-generation rate is too low, then each packet 
TABLE IV

Summary of UMTS-Like PARAMETERS

\begin{tabular}{|c|c|c|}
\hline \multirow[t]{2}{*}{ Feature } & \multicolumn{2}{|r|}{ Value } \\
\hline & BCH coding & Turbo Coding \\
\hline Modulation & \multicolumn{2}{|c|}{ Differential-QPSK } \\
\hline TDMA frame length & \multicolumn{2}{|c|}{$4.615 \mathrm{~ms}$} \\
\hline Slots/Frame & \multicolumn{2}{|c|}{8} \\
\hline Slot length & \multicolumn{2}{|r|}{$577 \mu s$} \\
\hline OFDM carricrs & \multicolumn{2}{|c|}{1024 (612 used +2 pilots $)$} \\
\hline System Bandwidth & \multicolumn{2}{|c|}{$1.6 \mathrm{MH} z$} \\
\hline System Symbol rate (symbols/sec) & \multicolumn{2}{|c|}{$2.17 \times 10^{6}$} \\
\hline Tormalised Doppler Frequency & \multicolumn{2}{|c|}{$4.267 \times 10^{-5}$} \\
\hline Coded Bits/slot & \multicolumn{2}{|c|}{1224 bits } \\
\hline Feediback control bits & \multicolumn{2}{|r|}{27} \\
\hline H.263 Packetisation header bits & \multicolumn{2}{|r|}{11} \\
\hline $\begin{array}{l}\text { Channel Coding } \\
(\approx 1 / 2 \text { rate })\end{array}$ & $\begin{aligned} 4 \times & \operatorname{BCH}(255,131,18)+ \\
2 \times & \operatorname{BCH}(127,64,10)+ \\
& \operatorname{BCH}(63,30,6)\end{aligned}$ & $\begin{array}{l}\text { Turbo Coding } \\
\text { using } 612 \text { bit random interleaver } \\
+16 \text { bit CRC }\end{array}$ \\
\hline Pre-FEC Bits per timeslot & 618 & 594 \\
\hline Pre-FEC Bitrate & $134 \mathrm{Kbit} / \mathrm{s}$ & $129 \mathrm{Kbit} / \mathrm{s}$ \\
\hline Video bits por timeslot (FEC) & 580 & 556 \\
\hline Useful Video Bitrate (FEC) & $126 \mathrm{Kbit} / \mathrm{s}$ & $120 \mathrm{Kbit} / \mathrm{s}$ \\
\hline
\end{tabular}

contains a high number of macroblocks, and therefore when a transmission packet is corrupted, a large proportion of the video frame is lost. Therefore, the packet-generation rate for each video resolution was adjusted experimentally, taking into account that as the video resolution was increased four-fold, corresponding to increasing the video resolution, for example, from QCIF to CIF, the number of macroblocks per TDMA frame or per time unit was increased by the same factor, resulting in a corresponding increase in terms of the packetgeneration rate. These aspects were discussed in the context of defining the FER at the end of Section III-A.

Again, the packet-generation rate for each of the four video resolutions used is shown in Table III in terms of the number of TDMA frames between video packets or, synonymously, the TDMA packet separation, as well as in terms of packets per second, which are matched to the bit-rate requirement of each mode. For example, for QCIF-resolution video, the packet-generation rate is 195 per second, which corresponds to one packet every 30 TDMA frames. After setting the packetgeneration rates for each video mode, the video target bit rates were set to give high quality for the majority of video sequences. As mentioned previously, the OFDM system can transmit 1022 bits in every time slot. Hence for the QCIF mode, with one time slot every 30 TDMA frames, the channel bit rate would be $1022 \times 195=200 \mathrm{Kbit} / \mathrm{s}$. Upon using halfrate channel coding, the video bit rate is constrained to 100 $\mathrm{Kbit} / \mathrm{s}$. Since we required around $500 \mathrm{Kbit} / \mathrm{s}$ for high-quality QCIF video for a wide range of video sequences, we decided to use five time slots once every 30 TDMA frames. Therefore, the channel bit rate became $5 \times 1022 \times 195=1 \mathrm{Mbit} / \mathrm{s}$, providing a bit rate of $500 \mathrm{Kbit} / \mathrm{s}$ for video source coding. ${ }^{2}$

For the WATM investigations, we decided to use embedded binary BCH block coding [41], since it is capable of both error correction and error detection. For all the modes, we used the

\footnotetext{
${ }^{2}$ The interested reader is referred to http://www-mobile.ecs.soton.ac. uk/peter/robust-h263/robust.html for some examples of coded sequences, which can be viewed using an MPEG player.
}

near half-rate code of $\mathrm{BCH}(255,131,18)$. The corresponding pre-FEC bit rates for the various modes are shown in Table III. In conjunction with this channel coding scheme, the pre-FEC bit rate for the QCIF mode is $511 \mathrm{Kbit} / \mathrm{s}$.

The videophone system requires some additional overhead for its operation, since the feedback information for the reverse link is concatenated with the information packet, requiring a maximum of 29 additional bits per packet. In addition, the H.263 packetization adds a header to each packet [37], which is dependent on the number of bits in each packet; for our system, this header was between 12-14 bits per packet. Therefore, the number of useful video source bits in each packet used for video transmission was about 40 bits less than the actual number of bits per packet. The corresponding useful video source bit rate for each of the modes is shown in Table III, which was $503 \mathrm{Kbit} / \mathrm{s}$ for QCIF-resolution video, when taking into account the above-mentioned transmission overheads.

\section{B. Video Parameters of the UMTS Scheme}

Our UMTS-type scheme was also designed for a range of bit rates and services. We opted for using the so-called highbit-rate slot type, of which there can be a maximum of eight in each TDMA frame. The OFDM system designed for this scenario gave 1224 bits per time slot, which yields a channel bit rate of $265 \mathrm{Kbit} / \mathrm{s}$ or approximately $130 \mathrm{Kbit} / \mathrm{s}$ before half-rate FEC coding. This bit rate is suitable for high-quality QCIF video or lower quality CIF video. Hence we limited our investigations to QCIF resolution. Additionally, we invoked two different types of channel coding, BCH block codes [41] and turbo coding [42], which were also specified in Table IV. Since our system also required an error-detection facility, the use of block codes is convenient due to their inherent error correction and detection capabilities. The parameters for the UMTS-type scheme are summarized in Table IV. Since turbo coding cannot provide error detection, to this effect a 16-bit cyclic redundancy checking (CRC) code was used. 


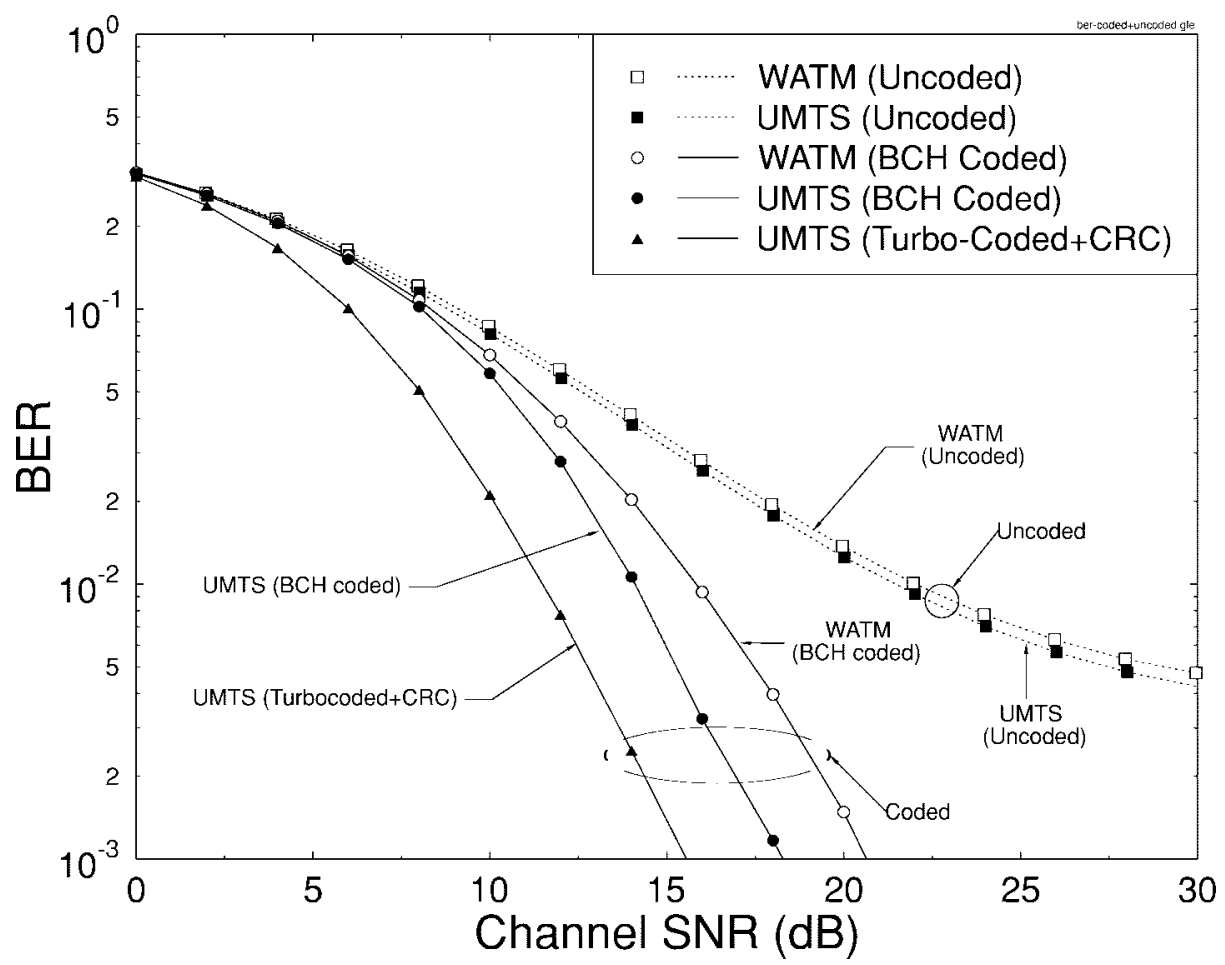

Fig. 13. Uncoded channel BER and channel-decoded BER of the WATM and UMTS systems over the wide-band channels characterized by the impulse responses shown in Figs. 10 and 11, respectively.

Given the 1224-bits/slot payload per TDMA frame, before channel coding the number of bits per TDMA frame was constrained to 618 for $\mathrm{BCH}$ coding and 594 for turbo coding. Half-rate turbo coding was used; however, two termination bits per slot were required for the convolutional encoders. Therefore, the number of pre-FEC bits per transmission packet was $1224 / 2-2$ (termination) $-16(\mathrm{CRC})=594$. This led to a pre-FEC bit rate of $134 \mathrm{Kbit} / \mathrm{s}$ for $\mathrm{BCH}$ coding and $129 \mathrm{Kbit} / \mathrm{s}$ for turbo coding. The additional system overhead required 27 bits per packet for the reverse link's acknowledgment flag and 11 bits for the H.263 packetization header [37]. This led to a video bit rate of $126 \mathrm{Kbit} / \mathrm{s}$ for $\mathrm{BCH}$ coding and 120 $\mathrm{Kbit} / \mathrm{s}$ for turbo coding. Having highlighted the salient videospecific system features, let us consider the achievable system performance in the next section.

\section{SYSTEM PERFORMANCE}

Fig. 13 portrays the BER performance of both candidate systems over the wide-band channels characterized by the impulse responses shown in Figs. 10 and 11, respectively. It is interesting to observe that since the number of subcarriers was sufficiently high in both systems for narrow-band subchannel conditions to prevail, the modem BER curves are fairly similar, irrespective of the different Doppler frequencies. Nevertheless, the slightly lower uncoded BER of the UMTS-type scheme manifested itself in a further improved FEC-decoded BER. Last, as expected, the similar-rate turbo codec outperformed the $\mathrm{BCH}$ codec in terms of BER.

Fig. 14 portrays the FER and the feedback error rate (FBER) performance of both systems. Despite the BER differences of the systems, their FER performances are fairly similar.
This indicates that the UMTS-like scheme's lower average BER actually results in a similar FER, despite its lower inburst BER, when a BCH code word was overwhelmed by an excessive number of channel errors. However, the lowest inburst BER of the turbo codec translated into a substantially reduced acknowledgment flag feedback error rate after MLD decoding.

Fig. 15 shows the video quality in terms of the average PSNR versus the channel SNR for QCIF-resolution video transmitted over the WATM scheme. The figure shows the video quality of a range of video sequences from the highly motion active Foreman and Carphone sequences to the lower activity Miss America sequence, which is more amenable to compression. For all the video sequences the PSNR starts to drop when the channel SNR falls below about $20 \mathrm{~dB}$. Due to lack of space, the FER versus the channel SNR (CSNR) performance of the system is not explicitly characterized in this paper, but our records show that the frame error rate around this CSNR value is about 3\%. The corresponding visual quality appears unimpaired, and the effects of dropping do not become evident for CSNR's in excess of $16 \mathrm{~dB}$. At $16 \mathrm{~dB}$, the frame error rate is $17 \%$. However, the effects of this packet loss are only becoming "just noticeable" at a CSNR of 16 $\mathrm{dB}$. The effect of the packet loss is that parts of the picture are "frozen," but usually for only one video-frame duration of about $30 \mathrm{~ms}$ at $30 \mathrm{fps}$, which is not sufficiently long for these artifacts to become objectionable. However, if the part of the picture that was lost contains a moving object, the effect of the loss of the packet becomes more obvious. Therefore, for more motion-active sequences, the effect of packet loss is more pronounced. 


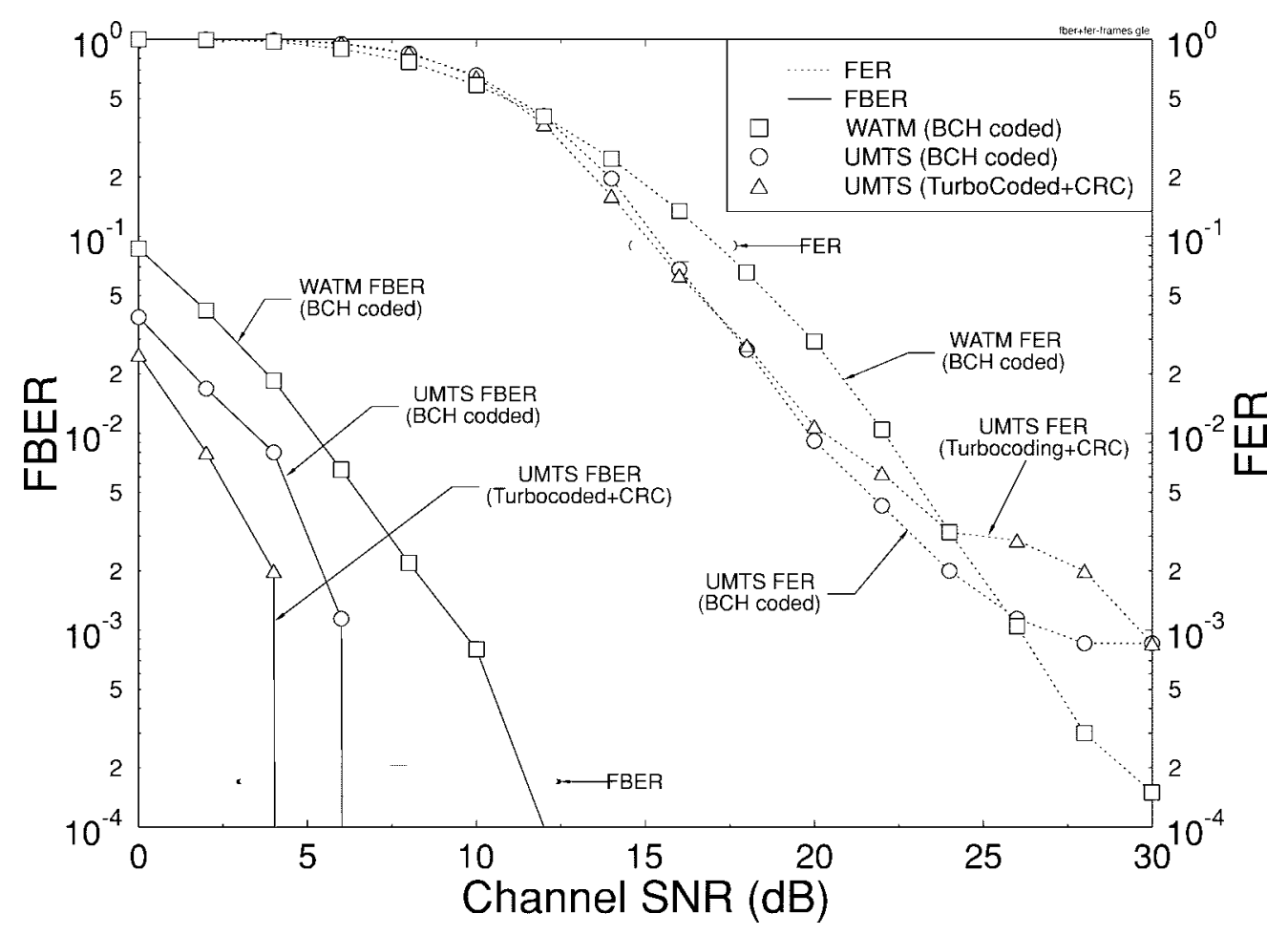

Fig. 14. FER and FBER after channel decoding versus channel SNR for the WATM system using BCH codes and for the UMTS-type scheme using $\mathrm{BCH}$ codes or turbo coding plus CRC over the wide-band channels characterized by the impulse responses shown in Figs. 10 and 11 , respectively. The WATM results are typical and are shown for the CIF mode of operation.

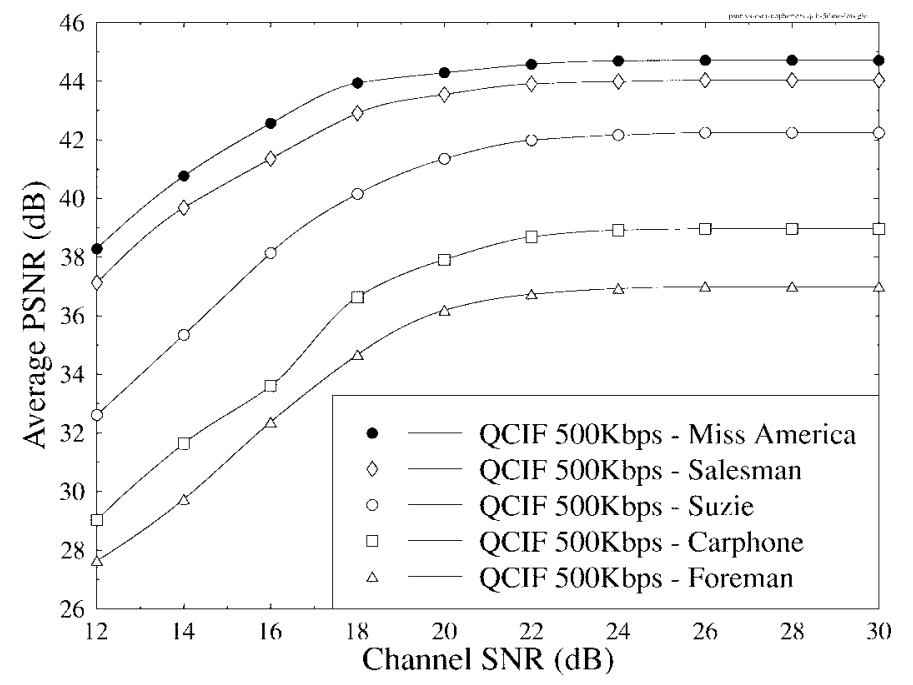

Fig. 15. QCIF video quality in PSNR versus CSNR at $500 \mathrm{Kbit} / \mathrm{s}$ and 30 fps over the WATM system, for various video sequences, using the impulse response of Fig. 10.

To portray the expected system performance in other application scenarios, where higher video quality is expected, in Fig. 16 we portrayed the average PSNR versus CSNR performance for a range of video resolutions from CIF to 16-CIF HDTV quality. At CIF resolution, the Miss America sequence was encoded at both $500 \mathrm{kbps}$ and 1 Mbps. For 4CIF resolution, the Suzie sequence was encoded at 2 and 10 Mbps, while for 16-CIF resolution, the Mall video clip was transmitted at 3 and 21 Mbps. This figure shows results for using multiple time slots per active TDMA frame, as suggested

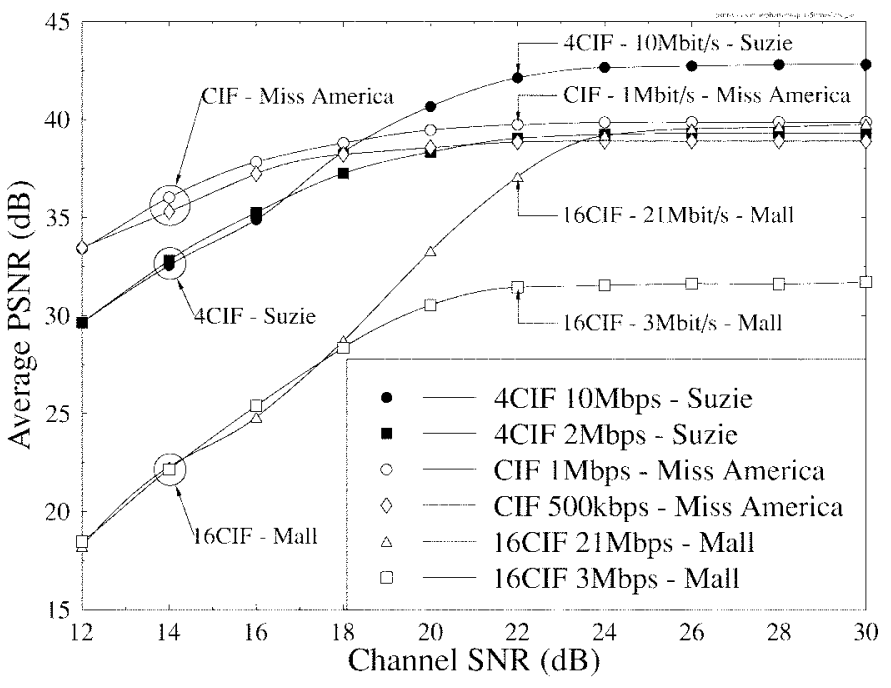

Fig. 16. Video quality in PSNR versus CSNR for CIF, 4-CIF, and 16-CIF resolution video at $30 \mathrm{fps}$ over the WATM system for various video sequences using the channel impulse response shown in Fig. 10.

by Table III, down to just a single time slot per active TDMA frame. Notice that the high- and low-bit-rate modes for each resolution seem to converge to a similar PSNR CSNR is low, which is a consequence of the higher PSNR degradations inflicted by a given FER in high-resolution modes due to their larger packet size, as seen in Table III and Fig. 8.

The 16-CIF scenarios seem to be more vulnerable to packet loss; however, this is because the packet-generation rate is not four times that of the 4-CIF simulations, and therefore each 16-CIF video packet contains approximately 2.5 times more 


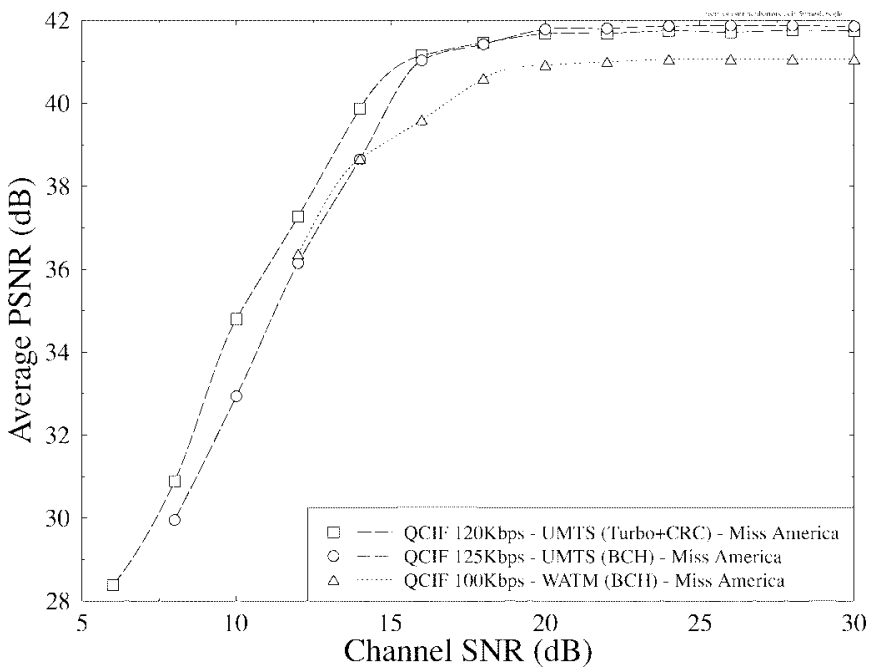

Fig. 17. Video quality in PSNR versus CSNR for the QCIF Miss America video sequence at $30 \mathrm{fps}$, using both the WATM system and the UMTS-type system with turbo coding and $\mathrm{CRC}$ or $\mathrm{BCH}$ coding, using the impulse responses shown in Figs. 10 and 11, respectively.

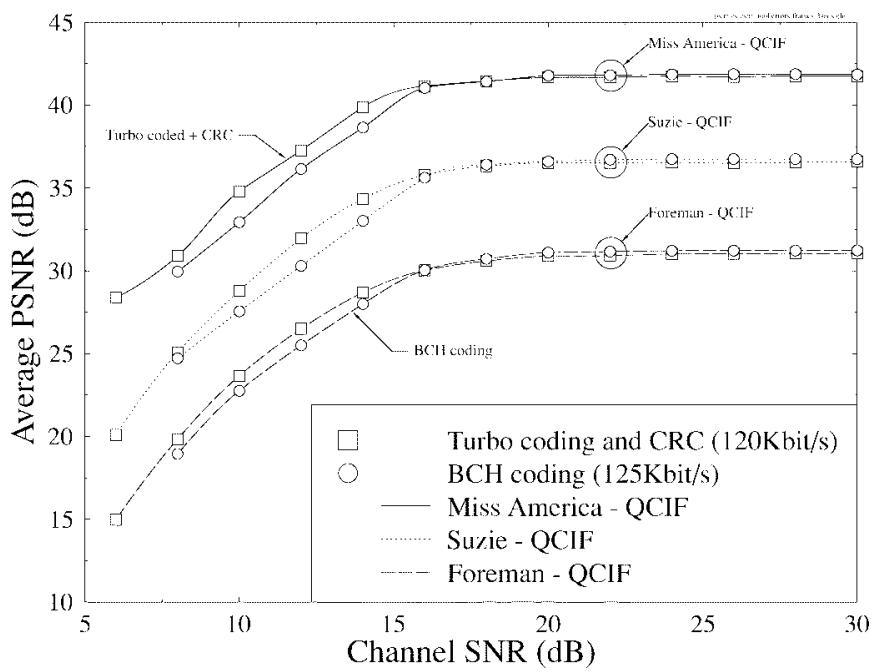

Fig. 18. Video quality in PSNR versus CSNR for a variety of QCIF-resolution video sequences at $30 \mathrm{fps}$ over the UMTS-type system with turbo coding and $\mathrm{CRC}$ or $\mathrm{BCH}$ coding using the COST 207 Bad Urban impulse response of Fig. 12 .

macroblocks per packet than the CIF and 4-CIF resolution video packets. Hence the effect of packet loss is more noticeable, and this is manifested in the faster reduction of the PSNR as the CSNR degrades.

Focusing our attention on the UMTS-like scheme, since the FER of the turbo coded scheme in Fig. 14 was not substantially lower than that of the $\mathrm{BCH}$ coded arrangement, the corresponding video PSNR performances are also quite similar, as evidenced by Figs. 17 and 18. This is a consequence of the system's high error resilience. Hence in practical systems, its added complexity may not be justified. A similarrate WATM performance curve is also shown in Fig. 17.

\section{CONCLUSION}

In this paper, the expected video performance of a WATM and that of a UMTS-type system was quantified in a variety of applications scenarios, using a range of video resolutions and bit rates. The high-efficiency H.263 video codec was employed to compress the video signal. The video formats used were summarized in Table III along with their associated target bit-rate figures. The proposed system ensures robust video communications using the WATM and the UMTS-type framework in a highly dispersive Rayleigh-fading environment, even at a vehicular speed of $30 \mathrm{mph}$, requiring channel SNR's in excess of only about $16 \mathrm{~dB}$ for near-unimpaired video transmission. Despite the different propagation conditions, the BER and FER modem performance of both systems was quite similar. Furthermore, due to the high error resilience of the video system, the increased complexity of the turbo codec was not justified in video performance terms, although the acknowledgment flag FBER was significantly reduced.

\section{ACKNOWLEDGMENT}

The authors wish to thank the EPSRC, U.K. They are indebted furthermore to J. Woodard for his kind assistance in the field of turbo coding and to the Median and First Consortium partners for fruitful discussions and for their friendship. The constructive criticism of the anonymous reviewers is gratefully acknowledged.

\section{REFERENCES}

[1] T. Ojanpera, "Overview of research activities for third generation mobile communications," in Wireless Communications-TDMA Versus CDMA, S. G. Glisic and P. A. Leppanen, Eds. Norwell, MA: Kluwer Academic, 1997, pp. 415-446.

[2] "Special issue on the European path toward UMTS," IEEE Personal Commun. Mag., vol. 2, Feb. 1995.

[3] K. Pehkonen et al., "A performance analysis of TDMA and CDMA based air interface solutions for UMTS high bit rate services," in Proc. PIMRC'97, Helsinki, Finland, Sept. 1997, pp. 22-26.

[4] A. Klein et al., "FRAMES multiple access mode $1-$ Wideband TDMA with and without spreading," in Proc. PIMRC'97, Helsinki, Finland, Sept. 1997, pp. 37-41.

[5] J. D. Gibbson, The Mobile Communications Handbook. New York: IEEE/CRC Press, 1996.

[6] Advanced Communications Technologies and Services (ACTS), European Commission, Workplan DGXIII-B-RA946043-WP, Aug. 1994.

[7] J. S. da Silva, B. Arroyo-Fernandez, B. Barani, J. Pereira, and D Ikonomou, "Mobile and personal communications: ACTS and beyond," in Wireless Communications-TDMA Versus CDMA, S. G. Glisic and P. A. Leppanen, Eds. Norwell, MA: Kluwer Academic, 1997, pp. 379-415.

[8] P. W. Baier, P. Jung, and A. Klein, "Taking the challenge of multiple access for third-generation cellular mobile radio systems-A European view," IEEE Commun. Mag., vol. 34, pp. 82-89, Feb. 1996.

[9] B. Engstroem and C. Oesterberg, "A system for test of multiaccess methods based on OFDM," in Proc. VTC'94, Stockholm, Sweden, 1994, pp. 1843-1847.

[10] W. Webb and L. Hanzo, Modern Quadrature Amplitude Modulation: Principles and Applications for Wireless Communications. New York: IEEE Press/Pentech, 1994.

[11] K. Pajukoski et al., "Wideband CDMA test system," in Proc. PIMRC'97, Helsinki, Sept. 1997, pp. 669-673.

[12] F. Adachi et al., "Coherent DS-CDMA-Promising multiple access for wireless multimedia mobile communications," in Proc. IEEE ISSSTA'96, Mainz, Germany, Sept. 1996, pp. 351-358.

[13] A. S. Acampora, An Introduction to Broadband Networks. New York: Plenum, 1994.

[14] M. Schwartz, Broadband Integrated Networks. Englewood Cliffs, NJ: Prentice-Hall, 1996.

[15] J. Y. LeBoudec, "The asynchronous transfer mode: A tutorial," Comput. Networks ISDN Syst., vol. 24, 1992.

[16] A. Acampora, "Wireless ATM: A perspective on issues and prospects," IEEE Personal Commun. Mag., vol. 3, pp. 8-17, Aug. 1996. 
[17] "Special issue on HIPERLAN," Wireless Personal Commun. J., vol. 3, no. 4 and vol. 4, no. 1, pp. 341-453, 1997.

[18] Radio Equipment and Systems (RES), "High performance radio local area network (HIPERLAN)," type 1, functional specifications, ETSI final draft prETS 300652 .

[19] T. Keller, J. P. Woodard, and L. Hanzo, "Turbo-coded parallel modem techniques for Personal Communications," in Proc. IEEE VTC'97, Phoenix, AZ, 1997, pp. 2158-2162.

[20] T. Keller and L. Hanzo, "Orthogonal frequency division multiplex synchronization techniques for wireless local area networks," in Proc. Personal, Indoor and Mobile Radio Communications (PIMRC'96), Taipei, Taiwan, Oct. 15-18, 1996, pp. 963-967.

[21] R. W. Chang, "Synthesis of band-limited orthogonal signals for multichannel data transmission," Bell Syst. Tech. J., vol. 46, pp. 1775-1796, Dec. 1966.

[22] B. Hirosaki, "An orthogonally multiplexed QAM system using the discrete Fourier transform," IEEE Trans. Commun., vol. COM-29, pp. 983-989, July 1981.

[23] J. Cimini, "Analysis and simulation of a digital mobile channel using orthogonal frequency division multiplexing," IEEE Trans. Commun., vol. COM-33, pp. 665-675, July 1985.

[24] F. Mueller-Roemer, "Directions in audio broadcasting," J. Audio Eng. Soc., vol. 41, no. 3, pp. 158-173, Mar. 1993.

[25] I. Kalet, "The multitone channel," IEEE Trans. Commun., vol. 37, pp. 119-124, Feb. 1989

[26] M. Sandell, J.-J. van de Beek, and P. O. Börjesson, "Timing and frequency synchronization in OFDM systems using the cyclic prefix," in Proc. Int. Symp. Synchronization, Essen, Germany, Dec. 14-15, 1995, pp. 16-19.

[27] A. Netravali and B. Haskell, Digital Pictures: Representation and Compression. New York: Plenum, 1988.

[28] A. K. Jain, Fundamentals of Digital Image Processing. Englewood Cliffs, NJ: Prentice-Hall, 1989.

[29] N. S. Jayant and P. Noll, Digital Coding of Waveforms. Englewood Cliffs, NJ: Prentice-Hall, 1984.

[30] N. MacDonald, "Transmission of compressed video over radio links," Br. Telecom Technol. J., vol. 11, pp. 182-185, Apr. 1993.

[31] M. Khansari, A. Jalali, E. Dubois, and P. Mermelstein, "Low bitrate video transmission over fading channels for wireless microcellular systems," IEEE Trans. Circuits Syst. Video Technol., vol. 6, pp. 1-11, Feb. 1996.

[32] R. M. Pelz, "An unequal error protected $p \times 8 \mathrm{kbit} / \mathrm{s}$ video transmission for DECT," in Proc. IEEE Vehicular Technology Conf., 1994, pp. $1020-1024$.

[33] N. Färber, E. Steinbach, and B. Girod, "Robust H.263 video transmission over wireless channels," in Proc. Int. Picture Coding Symp. (PCS), Melbourne, Australia, Mar. 1996, pp. 575-578.

[34] "Video coding for low bitrate communication," ITU-T Recommendation H.263, Mar. 1996.

[35] H. Gharavi and M. H. Partovi, "Multilevel video coding and distribution for emerging broadband digital networks," IEEE Trans. Circuits Syst. Video Technol., vol. 6, pp. 459-469, Oct. 1996.

[36] P. Cherriman and L. Hanzo, "Power-controlled h.263-based wireless videophone performance in interference-limited scenarios," in Proc. IEEE Int. Symp. Personal, Indoor, and Mobile Radio Communications, Taipei, Taiwan, Oct. 15-18, 1996, vol. 1, pp. 158-162.

[37] _ "Programmable H.263-based wireless video transceivers for interference-limited environments," IEEE Trans. Circuits Syst. Video Technol., vol. 8, pp. 275-286, June 1998.

[38] J. Streit and L. Hanzo, "Vector-quantised low-rate cordless videophone systems," IEEE Trans. Veh. Technol., vol. 42, pp. 340-357, May 1997.

[39] L. Hanzo and J. Streit, "Adaptive low-rate wireless videophone systems," IEEE Trans. Circuits Syst. Video Technol., vol. 5, pp. 305-319, Aug. 1995.

[40] J. Streit and L. Hanzo, "Quadtree-based reconfigurable cordless videophone systems," IEEE Trans. Circuits Syst. Video Technol., vol. 6, pp. 225-237, Apr. 1996.

[41] K. H. H. Wong and L. Hanzo, "Channel coding," Mobile Radio Communications, R. Steele, Ed. London, U.K.: IEEE Press/Pentech, 1992, chap. 4, pp. 347-489.
[42] C. Berrou, A. Glavieux, and P. Thitimajshima, "Near Shannon limit error-correcting coding and decoding: Turbo-codes," in Proc. ICC, 1993, pp. $1064-1070$

[43] "Special issue on MPEG-4" IEEE Trans. Circuits Syst. Video Technol., vol. 7, pp. 1-256, Feb. 1997.

[44] L. Hanzo, P. Cherriman, and J. Streit, Modern Video Compression and Communications over Wireless Channels. New York: IEEE Press, to be published.

[45] J. Brecht and L. Hanzo, "Statistical packet assignment multiple access for wireless asynchronous transfer mode systems," in Proc. ACTS Summit'97, Aalborg, Denmark, Oct. 1997, pp. 734-738.

[46] M. Failli, "Digital land mobile radio communications COST 207," European Commission, EUR 12160 EN (1989) FS, 1989.

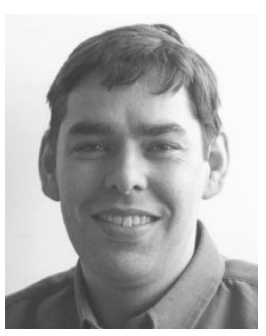

Peter Cherriman received the M.Eng. degree in information engineering from the University of Southampton, U.K., in 1994, where he currently is pursuing the $\mathrm{Ph} . \mathrm{D}$. degree in mobile video networking in the Department of Electronics and Computer Science.

$\mathrm{He}$ is working on projects for the Mobile Virtual Centre of Excellence, U.K. His current areas of research include robust video coding, microcellular radio systems, power control, dynamic channel allocation and multiple access protocols.

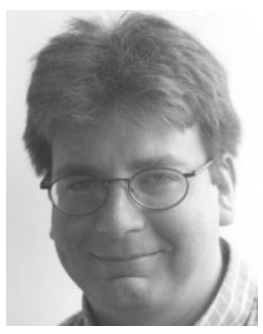

Thomas Keller studied Electrical Engineering at the University of Karlsruhe and Ecole Superieure d'Ingenieurs en Electronique et Electrotechnique, Paris, France. He received the Dipl.-Ing. degree from the University of Southampton, U.K., in 1995 , where he currently is pursuing the Ph.D. degree in mobile communications with the Wireless Multimedia Communications Group.

His current areas of interest include adaptive OFDM transmission, wide-band channel estimation, and error-correction coding.

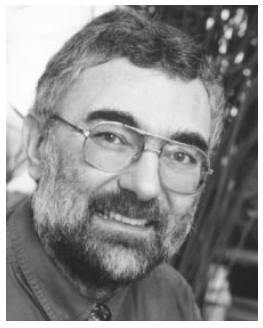

Lajos Hanzo (M'91-SM'92) graduated in electronics in 1976 and received the Ph.D. degree in 1983.

During his 20-year career in telecommunications, he has held various research and academic posts in Hungary, Germany, and the United Kingdom. Since 1986, he has been with the Department of Electronics and Computer Science, University of Southampton, U.K., and has been a Consultant to Multiple Access Communications Ltd., U.K. Currently, he holds a chair in telecommunications. He is a coauthor of three books on mobile radio communications. He has published more than 200 research papers, organized and chaired conference sessions, presented overview lectures, and been awarded a number of distinctions. Currently, he is managing a research team, working on a range of research projects in the field of wireless multimedia communications under the auspices of the Engineering and Physical Sciences Research Council, the European Advanced Communications Technologies and Services Programme, and the Mobile Virtual Centre of Excellence. 